\title{
A gestão educacional com o i-Educar: análise da viabilidade de implantação no município de Palotina - Paraná
}

\section{Educational management with i-Educar: analysis of the feasibility of deployment in the municipality of Palotina - Paraná}

\author{
Raquel Angela Speck ${ }^{1}$, Marcos Antonio Schreiner ${ }^{2}$, Josiane Patrícia Rodrigues dos \\ Santos Soares ${ }^{3}$, Lucas Bernardes da Silva ${ }^{4}$, Gabriel Augusto Lenhart ${ }^{5}$
}

\section{Resumo}

A sociedade moderna vive um modelo de organização política, cultural e administrativa em que a gestão eficiente das instituições públicas adquiriu centralidade e contornos gerenciais. No caso das instituições de ensino não é diferente, na medida em que a gestão escolar também tem sido discutida sob a ótica da qualidade e da eficiência. No contexto de desenvolvimento e evolução das novas tecnologias de informação e comunicação, a administração destas instituições requer pessoas que possam utilizar informações gerenciais para tomar decisões ágeis, assertivas, de qualidade e baixo custo. Tais requisitos quanto ao trato das informações podem ser alcançados mais facilmente quando se utilizam software gerenciais que automatizam o sistema de informação, monitoramento e controle. Neste artigo, analisamos a aplicação do software livre denominado i-Educar, que auxilia na gestão de informações educacionais, apontando para a viabilidade de sua implantação no município de Palotina, no Estado do Paraná. Após serem apresentadas e analisadas as suas principais características e funcionalidades, conclui-se pela adequação deste sistema à realidade educacional do município, dado a inexistência neste de programas para o planejamento e gestão dos dados educacionais.

Palavras-chave: i-Educar. Software educacional. Gestão educacional.

\begin{abstract}
Modern society has a model of political, cultural and administrative organization in which the efficient management of public institutions has acquired centrality and managerial contours. In the case of educational institutions it is not different, and school management has also been discussed from the point of view of quality and efficiency. In the context of the development and evolution of new information and communication technologies, the administration of these institutions requires people who can use managerial information to make agile, assertive, quality and low-cost decisions. Such requirements regarding the handling of information can be achieved more easily when using management software that automates the information, monitoring and control system. In this article, we analyze the application of free software called i-Educar, which assists in the management of educational information, pointing to the feasibility of its implementation in the city of Palotina, in the State of Paraná. After presenting and analyzing its main characteristics and functionalities, it concludes by the adequacy of this system

1 Pedagoga. Doutoranda em Educação. E-mail: raquel.speck@ufpr.br

2 Mestre em Informática pela Universidade Federal do Paraná. Professor assistente da Universidade Federal do Paraná.

3 Graduada em Gestão Pública pelo Instituto Federal do Paraná. Acadêmica do curso de Licenciatura em Computação da Universidade Federal do Paraná - Setor Palotina.

4 Acadêmico do Curso de Licenciatura em Computação, da Universidade Federal do Paraná - Setor Palotina.

5 Acadêmico do Curso de Licenciatura em Computação, da Universidade Federal do Paraná - Setor Palotina.
\end{abstract}


to the educational reality of the municipality being researched, given the inexistence in this program for the planning and management of educational data.

Keywords: i - Educar. Educational software. Educational management.

\section{Introdução}

A sociedade atual vive um modelo político, cultural e administrativo onde os ambientes formais de ensino, consubstanciados na instituição escolar, são convocados a adotarem e assumirem as novas tecnologias de informação e comunicação não apenas como ferramenta de ensino, mas também como recurso gerencial e administrativo. $\mathrm{O}$ movimento de reforma do aparelho administrativo do Estado, iniciado na década de 1990, destacou a necessidade de as instituições da administração pública tornarem-se menos burocráticas e adotarem uma postura mais gerencial, com foco no planejamento estratégico e nos resultados.

Com a redemocratização no final da década de 1980 e a Reforma do Estado na década de 1990, a estrutura federativa brasileira foi sendo transformada por meio de diversos processos, dentre os quais a descentralização das políticas públicas. Acompanhando essas mudanças, houve um movimento pela melhoria da gestão pública e valorização do planejamento introduzindo conceitos como eficiência (fazer mais com menos), eficácia (atingir metas e objetivos) e efetividade (resultados e impactos das ações estatais). Ao mesmo tempo, a democratização levou a uma maior pressão por participação social, transparência, prestação de contas e accountability na agenda governamental (OLIVEIRA; FALEIROS; DINIZ, 2015, p. 25).

Da escola, enquanto instituição pública de ensino, requer-se que, além de ter um corpo docente capacitado para promover a aprendizagem, disponham também de um corpo administrativo que garanta a continuidade, a eficiência e a eficácia da instituição. Este corpo administrativo precisa ser suprido com informações gerenciais precisas e adequado, muitas vezes provido por software.
De acordo com Oliveira (2013), os sistemas de informações nos setores escolares públicos têm uma importância ampla, pois é por meio deles que os planejamentos, as coordenações, avaliações e a produção de dados são implementados para as políticas públicas. Entretanto, algumas vezes as informações destes sistemas são generalistas e não há diálogo dos usuários com os gestores no processo de implementação, assim como não há interconexão entre os diferentes sistemas de informação públicos, dificultando as especificações das reais necessidades de cada unidade escolar ou de uma determinada região.

A necessidade de conhecimento especializado; no uso de tecnologias livres, emergentes e consolidadas para a gestão de instituições públicas de ensino é o principal elemento motivacional deste artigo. Na medida em que se verifica a existência de ferramentas tecnológicas livres, possíveis de serem implantadas em instituições públicas de ensino, tornam-se pertinentes as pesquisas voltadas a verificação da viabilidade de implantação destas ferramentas.

De acordo com o Portal do Software Público Brasileiro (2007), o i-Educar (Sistema Integrado e Centralizado de Gestão Escolar) é um software livre de gestão escolar que centraliza as informações de um sistema educacional municipal, diminuindo o uso de papel e a duplicidade de documentos. Ele foi desenvolvido inicialmente pela prefeitura de Itajaí-SC e tornou-se o primeiro programa a ser disponibilizado por uma prefeitura no Portal do Software Público Brasileiro. Com ele é possível controlar todo o cadastro de alunos, como seus dados pessoais, familiares e pedagógicos. Dentre suas funcionalidades destacam-se a gestão das matrículas, o uso do espaço das salas e o acompanhamento do aluno. Aos familiares é possível acompanhar pela 
Internet o desempenho dos alunos na escola, bem como comunicar-se com a escola.

No Paraná, atualmente a maioria das escolas municipais e estaduais utilizam o SERE (Sistema Estadual de Registro Escolar). Este sistema é implementado com base em software livre, mas é de propriedade da CELEPAR (Companhia de Informática do Paraná). Por meio dele, se padronizou a emissão de documentos, cadastros de alunos, matrículas, movimentações discentes e avaliações. Não pretendemos negar a sua contribuição para o gerenciamento e a padronização das informações e dos dados dos alunos, nem tampouco minimizar a sua capacidade de organizar o planejamento da instituição escolar e a efetivação da construção da vida escolar do aluno (OLIVEIRA, 2013). No entanto, pelo presente artigo, buscamos descrever o i-Educar, e compará-lo com o SERE. O objetivo é responder a seguinte pergunta: Um software livre para a gestão de escolas, como o i-Educar, possui as funcionalidades requeridas para suprir as necessidades sistêmicas de escolas da rede pública de ensino? Como delimitação do espaço, optamos em adotar como campo investigativo a rede pública municipal de Palotina, na região Oeste do Estado do Paraná. A coleta de dados foi realizada por meio entrevistas e questionários aos professores, equipe pedagógica e diretiva ${ }^{6}$.

\section{Histórico}

O i-Educar foi criado originalmente pela Prefeitura de Itajaí (SC), pelo Centro Tecnológico de Informação e Modernização Administrativa (CTIMA). Foi lançado em outubro de 2008 durante a Conferência Americana de Software Livre, a Latinoware. Uma primeira versão revisada foi disponibilizada em março de 2009.
Depois disso, o i-Educar já foi adotado em diversos municípios brasileiros, mas nenhum deles é no Paraná. Dentre os municípios que adotaram o i-Educar, destacamos o de Arapiraca, no Estado de Alagoas, que não somente utiliza o software em sua gestão educacional, mas também já contribui para a melhoria do mesmo a partir de feedbacks estabelecidos com a BB Tecnologia (antigamente conhecida como Cobra Tecnologia) desenvolvedora do projeto. O Estado de Alagoas, por outro lado, optou por criar um fork (uma espécie de bifurcação realizada a partir de um software já existente), a partir do qual conseguiu construir o seu próprio sistema de informação escolar, independente das novas versões do i-Educar (SOFTWARE PÚBLICO, 2016).

O i-Educar também foi usado como piloto para a cidade de Viçosa, no Ceará, sendo o primeiro a rodar após a montagem da cidade digital, no Projeto Viçosa Digital, que tem trabalhado no sentido de desenvolver e ampliar a infraestrutura de informação e comunicação no município. A secretaria de Logística e Estratégia Administrativa de Viçosa, Luciana Vieira, relatou que a implantação do i-Educar traria vários benefícios à gestão educacional, tais como, a geração de relatórios de frequência, diários de classe, notas e planos de aulas (RODRIGUES, 2015).

Uma das vantagens que se pode destacar com a utilização do i-Educar (assim como nos demais software livres) é a economia que ele proporciona para os municípios que o adotam. O município de Caçador, em Santa Catarina, por exemplo, registrou uma economia de $\mathrm{R} \$ 250.000,00$ após a sua implantação. Antes disso utilizava um software proprietário, isto é, privado e com direitos exclusivos para o desenvolvedor. No Distrito Federal estimase que a economia seja de R\$ 91 milhões desde 2012, quando o i-Educar foi implantado e acabou

\footnotetext{
${ }^{6}$ A pesquisa aqui apresentada resulta do projeto intitulado "Pesquisa da viabilidade de aplicação e uso de software livres para gestão em instituições públicas de ensino", inscrito na modalidade Licenciar da UFPR, Setor Palotina, iniciado em 2016 e finalizado em 2017, sob a coordenação do professor Marcos Antonio Schreiner e colaboração da professora Raquel Angela Speck. A pesquisa contou com o financiamento do programa Licenciar da UFPR, por meio de bolsas concedidas aos acadêmicos participantes do projeto.
} 
beneficiando mais de 448 mil estudantes e 640 escolas (PORTAL BRASIL, 2015).

\section{Funcionalidades do i-Educar}

O i-Educar é um software totalmente online que permite centralizar as informações das unidades escolares, interligando-as a um banco de dados único. Por meio dele é possível efetuar ações diversas, em tempo real, como cadastro de alunos, matrículas, transferências, emissão de certificados e diplomas, suspensões e geração de horários (PORTABILIS, 2016). A centralização dos dados e a geração de relatórios facilita e agiliza a tomada de decisões para os gestores, tanto na unidade escolar como na secretaria municipal. É possível detectar e solucionar um problema em qualquer ponto da rede de ensino e, se for o caso, dar-lhe o tratamento devido.

Para os pais e os alunos há funções interessantes, como a matrícula online e o acompanhamento de notas e frequência. Para este acesso é necessária a realização de um cadastro, que pode ser feito também online, a partir do qual será possível emitir boletins escolares, consultar ocorrências, acessar o quadro de avisos e também enviar mensagens ou e-mails. Ou seja, o i-Educar é também um importante canal de comunicação entre a escola e os pais. De acordo com informações do site Portabilis (2016), "o módulo Pais e Alunos funciona em conjunto com o i-Educar e permite que pais ou responsáveis e alunos consultem via web informações da vida escolar do aluno em toda a rede de ensino" e "oferece compatibilidade com todos os navegadores e dispositivos móveis como smartphones, tablets e notebooks". Logo, observa-se que tal funcionalidade auxilia os trabalhos da secretaria, na medida em que a maioria das informações podem ser obtidas via web, de qualquer lugar, e por meio de qualquer dispositivo conectado à web.

Integrado ao software há um sistema para gerenciamento de bibliotecas, que pode ter registro de autores, coleções e editoras. Por meio dele é possível o gerenciamento de empréstimos, devoluções e dívidas e pode ser feito por cada escola separadamente ou pelo município.

Por esses e outros motivos o i-Educar vem sendo recebendo cada vez mais visibilidade e espaço. Contudo, ainda assim, até o momento de nossa pesquisa, nenhum município do Paraná o está utilizando. É neste cenário que introduzimos nosso estudo, propondo a sua experimentação pela rede municipal de Palotina - Paraná.

\section{Considerações sobre o SERE - Sistema Estadual de Registro Escola}

A implantação do Sistema Estadual de Registro Escolar - SERE ocorreu em 1993, sendo lançado para o Sistema Operacional MS-DOS numa época em que as escolas não eram informatizadas. De acordo com Breda (2013, p. 96), a necessidade de um sistema capaz de armazenar as informações relacionadas aos alunos e de contribuir com a interligação dessas informações fez com que o SERE fosse desenvolvido, através de uma parceria com a Companhia de Tecnologia da Informação e Comunicação do Paraná (CELEPAR) e o Instituto de Desenvolvimento Educacional do Paraná (FUNDEPAR).

A primeira versão do SERE era offline e comportava um conjunto básico de funcionalidades. Como as escolas da época usavam sistemas manuais de informação, qualquer automatização, mesmo que mínima, era vista com interesse. Deste modo até mesmo as escolas particulares começaram a utilizar este sistema de gestão, que foi atualizado para o SERE-Web em 2005. Este sistema opera em software livre e está disponível para praticamente todas as escolas do Paraná (OLIVEIRA, 2013).

O funcionamento do SERE possibilitou aos Núcleos Regionais de Educação ter acesso aos sistemas das escolas, o que favoreceu a padronização da emissão de documentos, cadastros de alunos, matrículas, movimentações e avaliações. Contribuiu 
também para que as informações de dados dos alunos e o planejamento da instituição fossem relacionados à construção da vida escolar do aluno. A estrutura do SERE integra as áreas do sistema de registro escolar, planejamento escolar, estrutura e funcionamento da escola, de tal forma que:

A gestão dos dados permeia todo o processo pedagógico, do início ao fim, desde validar a entrada do aluno na escola, ao registrar sua permanência e desempenho em cada disciplina cursada, até o armazenamento e emissão de documentos relativos à história de escolarização do aluno. O SERE, a partir da secretaria de escola, permite a emissão de documentos padronizados dos estudantes e de relatórios referentes aos dados processados, integradas as áreas designadas como registro escolar, planejamento escolar, estrutura e funcionamento. Uma ação obrigatória do secretário ou auxiliar, além das mencionadas, corresponde à coleta de dados para o Censo Escolar pela atualização do cadastro da escola, funcionários, docentes, turmas e uso de transporte escolar atendem aos requisitos do sistema educacional (OLIVEIRA, 2013, p. 97).

Embora sejam inegáveis as possibilidades criadas pelo SERE na informatização da gestão escolar, existem algumas lacunas, que tanto as equipes da secretaria como as equipes pedagógicas destacam para o aprimoramento dos relatórios. Aponta-se a necessidade de alguns ajustes, que permitiram o aprimoramento no trato das informações, de forma que elas reflitam o perfil do aluno de forma satisfatória. Esses ajustes contribuiriam de forma significativa para melhoria do atendimento a todas as partes interessadas, conforme relato a seguir:

Registros de atendimento do estudante como ocorrências (poderiam ser enviadas aos pais), situação médica, comportamento do estudante, breve relato do Conselho de Classe, motivo de queda de aprendizagem, curso/disciplinas e estágio/área concluídos, atendimento de estudantes da Educação Especial, dados dos Diários de Classe do professor, o que representa atualização contínua, com acesso possível aos pedagogos (OLIVEIRA, 2013, p. 115).

A respeito desta informação, Oliveira (2013) informa que a SEED demonstra-se favorável ao aperfeiçoamento desses requisitos. Entretanto a lentidão nessa área requer que a equipe pedagógica busque soluções paralelas para sanar as lacunas existentes, como por exemplo, manter um cadastro paralelo para saber quais alunos são beneficiários do bolsa-família.

\section{O Acesso à Informação como Democratização da Gestão Educacional}

A informatização dos processos envolvidos na gestão educacional é sem dúvida uma forma de democratizar as relações entre escola e comunidade, especialmente quando os meios adotados possibilitam a interação entre os sujeitos envolvidos. Uma gestão escolar democrática está diretamente associada a democratização do acesso à informação, na medida em que:

A educação é um processo organizado, sistemático e intencional, ao mesmo tempo em que é complexo, dinâmico e evolutivo, em vista do que demanda não apenas um grande quadro funcional, como também a participação da comunidade, dos pais e de organizações diversas, para efetiválo com a qualidade necessária que a sociedade tecnológica da informação e do conhecimento demanda (LÜCK, 2009, p. 19).

No contexto de uma sociedade sempre mais assentada em bases tecnológicas, requer-se $\mathrm{o}$ desenvolvimento e a utilização de ferramentas que possam responder satisfatoriamente ao grande fluxo de dados existentes e que ao mesmo tempo garantam a participação e a interação dos sujeitos. Neste sentido, sistemas de informação multiusuário e participativos representam uma boa resposta a esta necessidade. 
Sistemas de informação que possuem processos de negociação mais participativos costumam ter melhores resultados e são melhor apropriados por seus usuários (OLIVEIRA, FALEIROS, DINIZ, 2015, p.23). Além disso, o acesso às informações principais das escolas e das redes de ensino contribui para a ampliação da compreensão e uso mais eficiente dos indicadores oficiais, que por sua vez tendem a refletir em políticas públicas mais adequadas ao contexto educacional.

No documento Indicadores da Qualidade em Educação (INEP, 2004), elenca-se a "informação democratizada" como um aspecto fundamental na gestão das escolas. Por meio deste indicador procura-se identificar em que medida a direção consegue informar toda a comunidade escolar sobre os principais acontecimentos da escola e se as informações circulam de maneira rápida e precisa entre pais.

Ainformatização da gestão educacional, contudo, encontra seu maior sentido quando articula a gestão administrativa e a pedagógica. $\mathrm{O}$ gerenciamento de atividades administrativas é sem dúvida uma tarefa importante e necessária no âmbito de qualquer unidade escolar ou rede de ensino. Mas é na gestão pedagógica que a escola cumpre o seu objetivo maior: zelar pela garantia da aprendizagem e sucesso dos alunos. O i-Educar possui funcionalidades que estão voltadas para este aspecto, integrando informações sobre os alunos, suas famílias, sobre os professores, funcionários, notas, parecer pedagógico de conselhos de classe, relatório por turmas, dentre outros, que permitem o acompanhamento e a gestão da aprendizagem. Além disso, há caminhos de comunicação da escola com os pais e a comunidade escolar, como email e sistema de notificação via safety management systems (SMS), o que facilita e agiliza a comunicação em situações necessárias. O acesso a histórico de boletins escolares é viabilizado online, o que é extremamente importante para o acompanhamento dos pais.
A edição do Prêmio Nacional de Referência em Gestão Escolar de 2007, que pretende servir como “estímulo à melhoria do desempenho da escola e ao sucesso da aprendizagem dos alunos pela identificação e reconhecimento, como referência nacional, de estabelecimentos escolares que estejam desenvolvendo práticas eficazes de gestão" define, como critério de eficácia, as "práticas de organização, atualização da documentação, escrituração, registros dos alunos, diários de classe, estatísticas, legislação e outros, para um atendimento ágil à comunidade escolar e ao sistema de ensino" (CONSELHO NACIONAL DE SECRETÁRIOS DE EDUCAÇÃO, 2007)

Evidencia-se, desta forma, a importância em se garantir a melhor comunicação, informação e atualização possível na gestão dos sistemas de ensino. Neste sentido, os software utilizado deve contribuir para este objetivo.

\section{Para cada Realidade, uma Necessidade}

A informatização dos processos de gestão escolar tem adquirido cada vez mais espaço nas unidades e nas redes de ensino. Entretanto, não se pode deixar de considerar as peculiaridades de cada contexto, que transparecem em forma de diferentes necessidades. Neste sentido, é fundamental considerar que cada escola tem uma situação concreta, o que coloca, para cada uma, necessidades diferenciadas quanto ao processo de gestão. Em se tratando de gestão informatizada não é diferente. É necessário que o programa utilizado seja flexível, permitindo a modificação de campos e a inclusão de outros.

Conforme destacado, o i-Educar é um software livre com Licença Pública Geral (GPL), que permite a manipulação do sistema, viabilizando a adaptação do mesmo à realidade que o demanda. A rigidez no que diz respeito a esta manipulação indica uma séria limitação na medida em que ignora tais necessidades. 
Outro aspecto interessante a ser destacado, que garante a adequabilidade do software a cada contexto, é a possibilidade existente no i-Educar de elaboração de quadro de horários. Sabe-se que esta é uma tarefa bastante trabalhosa quando realizada manualmente, e que por esta razão muitas escolas acabam adquirindo software pagos. Contudo, sabendo-se que a condição financeira destas, na maioria dos casos, não é satisfatória, este gasto representa um ônus para a gestão. Além disso, existe também o campo "calendário letivo", que permite buscar informações a respeito de aulas extras, aulas programadas, reposição de aulas e datas não letivas, feriados nacionais e comemorações.

\section{Acompanhamento pedagógico do aluno}

De acordo com Lück (2009), a qualidade do ambiente escolar como um todo determina a qualidade do processo pedagógico da sala de aula, que por sua vez é determinada por uma série de cuidados. É importante que a gestão escolar possa equilibrar gestão administrativa e pedagógica, sendo que nesta o acompanhamento individual do desenvolvimento do aluno torna-se questão de primeira ordem. Existem alguns recursos contidos no i-Educar que viabilizam esta verificação, pelos quais é possível obter o espelho de notas e relatórios de leitura e escrita. Destacamos, dentre estes recursos, os seguintes (tais como aparecem no programa): acompanhamento da leitura, alunos com benefícios; alunos em exame; ata de resultado final; boletim escolar; controle de desempenho de alunos; demonstrativo de alunos defasados; diário de avaliações; Diário de Frequência; Espelho de Notas; Ficha de Leitura e Escrita; Histórico Escolar; Levantamento de Alfabetizados e não Alfabetizados; Professores; Professores por Disciplina; Quadro Curricular; Resultado Final.

Estas opções colaboram para que a equipe de gestão e os professores possam ter acesso aos resultados dos alunos de maneira fácil, rápida $\mathrm{e}$ atualizada, utilizando tais dados para compor ações pedagógicas preventivas ou de intervenção.

\section{Gestão de notas e avaliações}

Pelo i-Educar torna-se possível gerir todo o processo avaliativo das escolas. Pode-se inserir nele as características do sistema de avaliação realizar o cálculo da média final. A sistemática de avaliação contida nos componentes curriculares (disciplinas) também pode ser inserida, pois o sistema permite o gerenciamento de fórmulas adicionais. Pode-se apurar também a frequência do estudante, no cômputo geral ou por disciplinas. Há a opção de geração de pareceres descritivos com notas numéricas ou a partir de conceitos. Enfim, há uma gama de possibilidades de personalização que facilita o trabalho do gestor e do usuário.

\section{Comparativo Entre o SERE e o i-Educar}

Para apresentar nossos resultados de modo mais objetivo, realizamos uma análise comparativa entre o i-Educar e o SERE, com o objetivo de sistematizar as informações levantadas, organizando-as de modo a apresentar as características de um e de outro. 
Tabela 1 - Comparativo entre os sistemas i-Educar e SERE

\begin{tabular}{|l|l|l|}
\hline Funcionalidades do sistema & i-Educar & SERE \\
\hline Licença GPL & Sim & Não \\
\hline Boletim e Histórico Escolar On-line & Sim & Sim \\
\hline Reserva de Vagas de Alunos On-line & Sim & Não \\
\hline Ocorrências disciplinares de Alunos & Sim & Não \\
\hline Sistema de matrículas online & Sim & Sim \\
\hline Calendário Escolar & Sim & Sim \\
\hline Quadro de Horários e Avisos Escolar & Sim & Não \\
\hline Cadastro de alunos e tipos especiais de alunos & Sim & Sim \\
\hline Cadastro de Tipos de Usuários & Sim & Não \\
\hline Cadastro de Servidores e Funcionários & Sim & Não \\
\hline Cadastro de Rede de Ensino e Escola & Sim & Sim \\
\hline Infraestrutura da escola & Sim & Não \\
\hline Cadastro de cursos, turmas e matérias & Sim & Sim \\
\hline Tipos de avaliação & Sim & Não \\
\hline Cadastro de Benefícios (Renda Familiar) & Sim & Sim \\
\hline Sistema de notificação via SMS & Sim & Não \\
\hline Sistema de Transporte Escolar & Sim & Sim \\
\hline Sistema de Biblioteca de Livros & Sim & Sim \\
\hline Sistema de Controle de Merenda Escolar & Sim & Sim \\
\hline
\end{tabular}

*A tabela foi construída a partir da sistematização das pesquisas realizadas em todo o material levantado de um e de outro sistema. Fonte: Autores.

O comparativo entre os sistemas aponta para a existência de um número maior de funcionalidades no i-Educar. Algumas destas funções são menos importantes e necessárias, como por exemplo, as notificações via SMS. Neste caso, a não existência da funcionalidade não representa um problema para o usuário ou para o gestor, assim como não demanda outra ação equivalente específica. Isto é, a função simplesmente não existe, mas não compromete a atividade envolvida. Por outro lado, a ausência certas opções, como a de um comando que permita elaborar o quadro de horário, faz com que as escolas tenham que buscar alternativas fora do sistema. No caso do horário escolar, por exemplo, as escolas precisam contratar outro software próprio para isso, tendo em vista que não é uma tarefa sempre fácil de ser realizada. Este é um custo extra que poderia ser evitado se a funcionalidade existisse. Ademais, a ausência da licença GPL impede alterações no sistema e acréscimo de opções adicionais.

\section{Considerações Finais}

Considerando-se os dados obtidos até o presente momento a respeito das funcionalidades do i-Educar, consideramos que o mesmo pode proporcionar uma maior transparência e agilidade para a gestão dos sistemas educacionais. Alguns aspectos apontados neste texto, como o acesso das informações pelos pais ou responsáveis, o gerenciamento de notas, faltas, pareceres, relatórios, bibliotecas, transporte escolar entre outros, imprimem ao i-Educar as características que se esperam de sistemas de informações eficazes e ao mesmo tempo democráticos. 
O i-Educar é um sistema web, ou seja, pode ser acessado de qualquer lugar e por qualquer dispositivo móvel, tais como notebooks, tablets e smartphones. Deste modo pode-se proceder às reservas de vagas online, para garantir a matrícula na instituição, permitindo agilidade no processo e evitando filas e esperas na secretaria.

Além disso, destaca-se a questão da economia financeira que ele proporciona, visto que se trata de software livre. Ademais, por possuir código aberto permite alterações em seu sistema, de modo a configurá-lo a partir das necessidades do usuário.

Nesse sentido, nossa pesquisa permitiu avaliar que, pelas suas funcionalidades, o i-Educar é adequado às escolas da rede municipal de Palotina - PR e à secretaria municipal de educação. Acreditamos que a sua utilização poderá contribuir de maneira significativa ao processo de gestão educacional no município, trazendo benefícios para a gestão educacional local. Para tanto, embora as definições atuais do sistema já possibilitem a sua pronta utilização pelo município, um estudo posterior mais detalhado poderá identificar quais alterações específicas no i-Educar permitirá uma melhor adaptabilidade deste às demandas de cada unidade escolar bem como da Secretaria Municipal de Educação.

\section{Referências}

BREDA, S. M. Informação escolar: o sistema estadual de registro escolar (SERE) do Paraná. Revista LatinoAmericana de Inovação e Engenharia de Produção, Curitiba, v. 1, n. 1, jan./jun. 2013.

CONSELHO NACIONAL DE SECRETÁRIOS DE EDUCAÇÃO - CONSED. Manual de orientações do prêmio nacional de referência em gestão escolar. Brasília, 2007. Disponível em: <www.drearaguaina. com.br/gestao_pedagogica/premio_gestao_2007.doc $>$. Acesso em: 17 jan. 2018.

INEP. O Instituto Nacional de Estudos e Pesquisas Educacionais Anísio Teixeira Indicadores da qualidade na educação. São Paulo: Ação Educativa, 2004.
LÜCK, H. Dimensões de gestão escolar e suas competências. Curitiba: Positivo, 2009.

OLIVEIRA, C. M. C. Informação a serviço da escola: o sistema estadual de registro escolar. 2013. Dissertação (Mestrado em Ciência Gestão e Tecnologia da Informação) - Universidade Federal do Paraná, Curitiba.

OLIVEIRA, L. C. P.; FALEIROS, S. M.; DINIZ, E. H. Sistemas de informação em políticas sociais descentralizadas: uma análise sobre a coordenação federativa e práticas de gestão. Revista de Administração Pública, Rio de Janeiro, v. 49, n. 1, p. 23-46, fev. 2015.

PORTABILIS. Soluções para a gestão pública. Disponível em: <http://www.treinamento.ieducar.com. br/produto/educacao-19>. Acesso em: 27 jul. 2016.

PORTAL BRASIL. DF economiza R\$ 91 milhões com software público. 2015. Disponível em: <http://www. brasil.gov.br/governo/2015/04/df-economiza-r-91milhoes-com-software-publico>. Acesso em: 4 maio 2016.

RODRIGUES, J. (Colab.). Viçosa será primeira cidade a usar a plataforma iEducar. Diário do Nordeste, Fortaleza, 11 abr. 2015. Regional. Disponível em: $<$ http://diariodonordeste.verdesmares.com.br/cadernos/ regional/vicosa-sera-primeira-cidade-a-usar-aplataforma-ieducar-1.1265226>. Acesso em: 17 jan. 2018.

SOFTWARE Público. Disponível em: <https:// softwarepublico.gov.br/social/>. Acesso em: $27 \mathrm{jul}$. 2016. 
